\title{
Citation Practices of Expert French Writers of English: Issues of Attribution and Stance
}

\author{
Elizabeth Rowley-Jolivet and Shirley Carter-Thomas
}

\begin{abstract}
In research articles (RAs), reporting the results and claims of other authors is a crucial skill as it both demonstrates the writers' familiarity with the literature of the field and allows them to position their own findings and conclusions within the existing body of research, thus creating space to promote their work. Using citations effectively, however, demands considerable linguistic and rhetorical expertise. While several studies have shown that citation causes problems for novice researchers, the specific linguistic problems of expert non-English-speaking researchers have been little investigated. We hypothesize that their problems are unlikely to be the same as those of novices and that cultural and language factors may interfere when citing in a foreign language. To test this hypothesis, we collected a corpus comprising three subsets of articles: 40 pre-publication uncorrected draft manuscripts written in English by expert French researchers in engineering, science and computational linguistics; a comparable corpus of 40 published RAs by native English researchers in the same disciplines; and 40 published RAs written in French by French researchers. The drafts were first examined to detect potential problems with citation; we then checked whether these problems also occurred in the English RAs; if not, this was considered to indicate that it might be a problem specific to French researchers writing in English. The French RAs were then analysed to see which problems could be attributed to the influence of the French language or French citation conventions. The concordancer AntConc 3.2.1. was used for quantitative searches in the corpus. The results revealed that four features related to issues of attribution and stance were particularly problematic for the expert French writers: the use of reporting verbs, of according to, of the wouldconditional, and concessive if-clauses. The French writers of English used reporting
\end{abstract}

\author{
E. Rowley-Jolivet ( $\bowtie)$ \\ Laboratoire Ligérien de Linguistique, Université d'Orléans, Orléans, France \\ e-mail: elizabeth.jolivet@univ-orleans.fr \\ S. Carter-Thomas \\ Institut Mines-Télécom/TEM and LATTICE CNRS, Paris, France \\ e-mail: Shirley.Thomas@telecom-em.eu
}


that-clauses far less than the English writers, and with a more restricted range of verbs, a profile of use reflecting that of the French RA subset. The other three problems relate to the different spectrum of values that the English expressions and their French equivalents can take: according to and selon express different degrees of writer commitment to the cited source; the French conditional is widely used to express lack of commitment or distance, a value that is not directly transposable into the English would-conditional; si-clauses are frequently used to express concession, unlike if-clauses. French writers of English tended to import all these features specific to French into their English drafts, resulting in many cases of ambiguity as to the writer's position towards the cited source. This cross-linguistic study shows that citing in English is far from straightforward for writers of other languages, and that citation practices are neither language- nor culture-free. The influence of the writer's native language and of French academic citing conventions can be clearly perceived in the citing structures and strategies adopted, often leading to a lack of clarity in this respect and thus significantly weakening the strength of the argument.

Keywords Citation - Expert writers - French researchers - Attribution - Stance

\section{Introduction}

Reporting the results and claims of other authors is a crucial skill in positioning one's own research findings and conclusions. An effective use of citations enables academic writers to situate their work within an existing body of research, demonstrating their membership of the disciplinary community, and at the same time to create space to promote their own research (Swales 1986, 2004; Hyland 1999, 2002; Fløttum et al. 2006). Using citations appropriately however requires a considerable amount of disciplinary and rhetorical know-how as well as the writing skills to match. For this reason instruction and advice on citation practice often figure prominently in ESP courses (cf. Swales and Feak 2004, 2009).

Using in-text references and their associated linguistic conventions appropriately can be difficult for novice researchers, whether native or non-native speakers. Past research on novice research writing has noted that novice researchers frequently experience difficulty in incorporating references into their texts effectively and finding their own voice. In a study of reports by doctoral-level students in the French social sciences, Boch and Grossman (2002) for example noted a tendency among French students to overuse full quotations, rather than use paraphrasing or non-integral citation. In English, Thompson and Tribble (2001) also identified some recurrent problems among student writers such as a lack of variety of citation types (overuse of according to), an inappropriate selection of reporting verbs, and a relative absence of non-integral references. Mansourizadeh and Ahmad (2011) likewise note a lack of non-integral citations in the articles of novice writers. 
Whilst the citation practices of novice writers (native and non-native speakers) have been thoroughly investigated, the specific linguistic problems of expert non English-speaking researchers in this area have to the best of our knowledge received much less attention. Citing appears at first sight to be a relatively culturefree feature of research publications in that the systems used are international. ${ }^{1}$ Expert writers can also be expected to be familiar with journals' expectations; this does not however mean that citation use is easily transferrable from one linguistic and academic culture to another. Our hypothesis is that cultural and linguistic factors may interfere when researchers are obliged to or choose to write in another language, and that the problems of experienced researchers are not necessarily the same as those of novices. In this article we focus therefore specifically on the citation and referencing practices of experienced French researchers publishing journal articles in English.

A cursory comparison between French and English academic citation practice reveals few major differences. Experienced French researchers use citations in much the same way and in the same quantities as their English-speaking colleagues, when writing in English (see Sect. 2). They are obviously aware of the conventions and know what the expected frequency, place and functions of citations are. However our experience of rereading and editing our French colleagues' articles over the years has also alerted us to a number of specific problems relating to the way research claims are attributed and positioned, and where citations are arguably not being put to their best use.

A key issue, for the reader of a text but also for any system of data mining or opinion mining, is to be able to identify the enunciator of the textual segment: who is speaking - the author of the cited text, or the writer of the citing text? This is a conventional, even compulsory, requirement in research articles, where information needs not only to be accurately traceable but also clearly attributed, since the source of the information provides the grounds for its evidential status. A second important question is the degree of the writer's commitment to the text that $\mathrm{s} / \mathrm{he}$ is citing: is there full writer commitment to the cited text, or does the writer distance herself in some way?

To investigate these two questions, we will focus on four specific features which proved, from our experience of editing research chapters, to be problematic for expert French writers of English: reporting that-clauses, use of the would conditional, of according to, and concessive if-clauses. While these four features do not by any means exhaust all the citation problems encountered by expert French writers of English, they are particularly important aspects to address in an

\footnotetext{
1 Though the citation systems themselves are international, Lillis et al. (2010), looking at citation practices from a more critical, or geolinguistic perspective, argue that the pressure to publish in English in high impact factor journals brings with it the pressure to cite primarily Englishmedium publications, and that in this light, citation appears to be dominated by Anglophone cultures. This leads them to conclude that English cannot be viewed as a neutral medium, since "its status within global evaluation systems is actually shaping what gets counted as knowledge" (Lillis et al. 2010: 131).
} 
initial approach to this topic. Reporting verbs and according to are among the most common devices used in research articles to attribute results or claims to other authors and to express the writer's position with respect to these cited sources, and therefore play a prominent role in citation. An additional resource in French academic articles to express nuances of writer commitment to the cited results or claims is the conditional verb form. This resource is not transferable to English and raises problems for the French researchers when writing in English. A similar cross-linguistic difficulty is encountered with French si-clauses, frequently used in RAs to concede, and background, others' findings; due to semantic differences between French si and English if (Carter-Thomas 2007), French writers of English have difficulty engaging in concessive argumentation when referring to cited sources.

\section{Corpus and Methodology}

We collected three sets of articles for this study (see Table 1). The first subset comprises 40 pre-publication uncorrected draft manuscripts written in English by French academics (FWE). These drafts were drawn from our experience of editing research manuscripts and covered several fields (engineering, science, and computational linguistics). All these articles, once revised, were subsequently submitted for publication in international journals. The second subset consists of a comparable corpus of 40 published research articles (RAs) written by native English researchers (NS) in the same disciplines. Three criteria were used to assign native-speaker status: (i) the institutional affiliations of the authors were in English-speaking countries; (ii) the articles were written in fluent English with no language errors; (iii) all the cited publications were in English. The authors' first and last names provided additional confirmation of their English L1 status. Although not completely fool-proof, these criteria were felt to be sufficiently discriminating with respect to the FWE authors, all of whom are affiliated to French institutions, live in France, and on occasion cite publications in French. The third subset consisted of a corpus of 40 published RAs written in French by French researchers $(\mathrm{F})$, again in the same disciplines.

The rationale for structuring the corpus in this way was the following. We first examined the drafts to detect potential problems with citation and referencing.

Table 1 Corpus

\begin{tabular}{llcll}
\hline Authors & Category & Number & Tokens & $\begin{array}{l}\text { Citations per } \\
10,000 \text { words }\end{array}$ \\
\hline French writers of English (FWE) & Uncorrected drafts & 40 & 190393 & 102 \\
Native English writers (NS) & Published RAs & 40 & 287519 & 104 \\
Native French writers (F) & Published RAs & 40 & 243910 & 101.5 \\
Total & & 120 & 721822 & \\
\hline
\end{tabular}


Secondly, we checked whether these problems also occurred in the native English corpus; if not, this was considered to indicate that it might be a problem specific to French researchers writing in English. The corpus of French RAs was then used as a reference corpus to see which of the problems could be attributed to the direct interference of the French language or of French citation conventions, rather than to the idiosyncrasies of particular writers.

As the last two columns in the table show, although the native English corpus is larger than that of the French writers of English in terms of word count-287,000 words against 190,000 - the number of citations, expressed per 10,000 words, is practically identical: 102 and 104. Almost exactly the same frequency of citation was found in the French subset (101.5), indicating that whatever the language used, among confirmed researchers there is a remarkable stability in the citation ratio. This gave us confidence that, quantitatively speaking, the French writers of English could be considered to adopt the citation practices expected of expert writers.

It was not possible to do a full comparison of the distribution of the citations over the different sections of the articles, as not all the texts followed the classical IMRaD format: several science articles in both the FWE and NS subsets had merged Results + Discussion sections, and various article formats were encountered in some of the computational linguistics articles. From the comparable elements at our disposal, however, there appear to be few discrepancies in the distribution of citations between FWE and NS RAs: in both cases, the 40 Introductions account for a little over one-third of all citations (FWE: $35 \%$; NS: $36.5 \%$ ), while the Results section, in those articles where this constituted a separate section, accounted for a negligible percentage of citations in both subsets (FWE: $5.3 \%$; NS: $3.8 \%$ ). Both the number and distribution of citations seem, therefore, to clearly indicate that expert writers, whatever their native language, are familiar with journals' expectations and disciplinary practices in this respect.

The articles in the corpus made use of the two main referencing systems prevalent in scientific research articles today: the author-date (or Harvard) system and the number (or IEEE) system with or without author name. Some of the linguistics articles (and a small proportion of the science articles) used the author-date system:

Thus, Fabb and Halle (2008) argue that metres always determine the number of syllables in the line.

However the number system was generally predominant, particularly in the science and engineering articles:

Koros et al. [5] proposed a mechanism to...

For instance biaxial tensile tests have been developed [4][5][6][7].

Another specificity of science articles is the almost total absence of verbatim quotation. In the science and engineering part of our corpus, for example, there is only one full quotation and two brief quotes. 
In the remainder of this article we will focus on questions related to issues of attribution, commitment and writer stance, which proved from analysis of the corpus to be a recurrent problem. The first feature addressed is reporting that-clauses.

\section{Reporting Verbs}

Previous studies of research discourse have shown that reporting that-clauses are very widely used so as to clearly express attribution (e.g. Charles 2006; Hyland 2002; Thomas and Hawes 1994; Thompson and Ye 1991). Two verb patterns are called upon here: V-that (Brown argues that...), and it be V-ed that (It has been reported that....). Using the concordancer AntConc 3.2.1 (http://www.antlab.sci. waseda.ac.jp), a search was therefore carried out on the word that in the FWE and NS subsets, and all the occurrences of finite reporting clauses were identified. While this method does not pick up cases with that-deletion, it was found in fact that omission of the that complementizer was extremely rare: a back-search on the eight most frequent reporting verbs in the NS and FWE subsets (argue, assume, conclude, demonstrate, find, note, show, and suggest) detected only a further six occurrences, confirming Biber et al.'s finding that "retention of that is the norm in academic prose" (Biber et al. 1999: 680), also noted by Charles (2006: 312). In the French subset, the search term was the complementizer que; since que-deletion is not possible in modern French, the figures can be considered exhaustive.

Although the distinction between statements of general disciplinary knowledge (called 'general reference' by Charles 2006) and citation is not always clear-cut, we adopted the following inclusion and exclusion criteria: general knowledge statements that comprised no precise reference or citation, and that did not correspond to a particular school of thought or work of a researcher were excluded; cases in which the reference to the school of thought or researcher could be retrieved from elsewhere in the text and that were considered sufficiently explicit for specialists in the field, even in the absence of a reference in the sentence itself, were however included. The final figures are given in Table 2.

Taking just the NS and FWE figures first, there are two striking differences between these speaker groups in the use of reporting verbs. The first concerns the frequency with which reporting that structures are used: only 5.9 per 10,000 words in the FWE drafts, against 13.9 per $10,000 \mathrm{w}$. in the NS set. There are several possible explanations for this marked discrepancy. The relative underuse of reporting verbs by FWE could for example be attributed to lack of awareness of the relevant English structures for reporting, i.e. to insufficient mastery of the

Table 2 Reporting that-clauses

\begin{tabular}{llll}
\hline & Occurrences & Per 10,000 words & Number of different verbs used \\
\hline NS & 400 & 13.9 & 55 \\
FWE drafts & 114 & 5.9 & 30 \\
French & 130 & 5.3 & 25 \\
\hline
\end{tabular}


English language. Although language problems were encountered with other types of citation structures (see Sect. 4), a close examination of the occurrences revealed very few problems of this kind with reporting verbs, and competent citation passages such as the following were the general rule:

(1) It was previously thought that the ketonic function of a sphagnan monomer, the 5-ketomannuronic acid, was responsible for the Maillard like reaction. However, Ballance et al. (2007) showed that this monomer was actually only present in sphagnan as trace. Instead, they found strong indication that sphagnan contains O-acetyl functionalities (FWE)

Another possibility is that FWE writers have the same difficulties as novices in conveying nuances of evaluation and positioning when citing others' work (Thompson and Tribble 2001; Mansourizadeh and Ahmad 2011). As the quantitative indicators given in the previous section show, however, their citation practices fully conform to disciplinary expectations both in distribution and overall frequency. A more likely explanation is suggested by the figure in Table 2 for the French writer subset, which with a frequency of 5.3 occurrences per $10,000 \mathrm{w}$. is almost identical to that of the FWE writers (5.9). The general frequency of reporting that structures for citation in the two languages appears from the present data to differ sharply (almost 3 times higher in NS than in F), probably indicating that other types of citing structures are used in French academic discourse, and that the FWE writers have carried this feature of their native language culture over into their writing in academic English.

Our aim here was not to analyze in detail the French subset, but some of these other structural types can be briefly mentioned: as demonstrated by Charolles and others (Charolles 2005; Charolles and Péry-Woodley 2005), the use of sentenceinitial discourse-framing devices is a recurrent feature of French discourse, and we speculate that this may be one of the explanations for the much lower use of reporting that/que structures. Example (2) illustrates this feature.

(i) Preference in French for an introductory evidential adverbial (D'après X, Pour $X$ et $Y$, Selon les résultats de $Z$ ) followed by a main clause, rather than a dependent clause after a reporting verb:

(2) Pour Haddock et al. [15], l'irradiation partielle de l'encéphale est associée à une augmentation du nombre des rechutes cérébrospinales. (F)

(For Haddock et al. [15], partial irradiation of the brain is associated with an increase in the number of cerebrospinal relapses. In preference to: Haddock et al. [15] argue/found that partial irradiation...)

(ii) The use of a nominal complement in French, where English traditionally prefers a clausal complement (Chuquet and Paillard 1987), likewise results in the absence of a reporting verb, as in example (3):

(3) L'expérience de l'Institut Gustave-Roussy [15] a également montré des taux de conservation élevés de (...) (F)

(The Institut Gustave-Roussy data also showed high rates of conservation of (...)

In preference to: The Institut Gustave-Roussy data also showed that the conservation rates of (...) were high) 
This deserves further study, but the consequence of these cross-linguistic differences and the subsequent scarcity of reporting verbs in the FWE drafts is that the French writers of English do not appear to position themselves as distinctly as the native English writers do.

The second difference clearly shown by Table 2 concerns lexical variety: a much smaller range of verbs (30) is used in the FWE subset- a figure that is again very similar to that of the French RAs (25) — compared to the NS group (55). Twothirds $(35 / 55)$ of the verbs used by the native English writers are not used at all by the French writers; a few others are much less used by the FWE writers; while conversely, 12 of the 30 verbs used by FWE are not used by the NS writers (see Table 3).

While the absence of some of the verbs in column 1 (e.g. confirm, demonstrate, indicate, remark) in the FWE subset may reflect the incomplete coverage of our corpus, the absence of some others (e.g. advocate, document, entertain, maintain, posit, postulate, speculate, state), we feel, indicates the French writers' reliance on a small set of verbs in reporting-that structures. The lexical range called upon does not include these more rarely-used verbs, although all are of Latin origin and so presumably familiar to French writers. Again, this may reflect the influence of their native language and academic culture, since both the FWE and F subsets rely very heavily on one verb, show/montrer, which accounts for 39 and $34 \%$ of all occurrences respectively, compared to only $18 \%$ in the NS subset.

Column 2 (verbs much less used by FWE than by NS) lists what could be called the 'staple' reporting verbs in NS articles: these eight verbs account for half (49\%) of the total in the NS articles, compared to less than a quarter in FWE. The very infrequent use of argue and claim, in particular, is rather surprising, since the expert French writers, unlike novice writers (cf. Introduction), do engage in cogent argument and confrontation of different points of view. This does not appear to be accomplished primarily, however, by using the reporting-that structures that are expected in English. The resulting effect on the reader is that nuances of stance are not explicitly conveyed, making it harder to grasp the writer's positioning towards the cited authors.

Table 3 Comparison of reporting verbs used by NS and FWE

\begin{tabular}{|c|c|c|}
\hline Verbs used by NS but not by FWE & $\begin{array}{l}\text { Verbs used much less } \\
\text { by FWE than by NS }\end{array}$ & Verbs used by FWE but not by NS \\
\hline 35 & 8 & 12 \\
\hline $\begin{array}{l}\text { Accept, acknowledge, advocate, } \\
\text { assert, believe, comment, } \\
\text { confirm, declare, demonstrate, } \\
\text { document, entertain (the idea } \\
\text { that), estimate, expect, explain, } \\
\text { feel, indicate, insist, maintain, } \\
\text { note, posit, postulate, predict, } \\
\text { present, protest, put, recognize, } \\
\text { remark, reveal, seem, speculate, } \\
\text { state, stress, support, take, write }\end{array}$ & $\begin{array}{l}\text { Argue, assume, claim, } \\
\text { conclude, find, } \\
\text { propose, say, } \\
\text { suggest }\end{array}$ & $\begin{array}{l}\text { Admit, advance, affirm, announce, } \\
\text { consider, emphasize, establish, } \\
\text { highlight, prove, suspect, think, } \\
\text { underline }\end{array}$ \\
\hline
\end{tabular}


The last column in Table 3 lists the verbs found only in the FWE subset. Some of these clearly show the influence of French lexis: advance that (Fr. avancer, instead of put forward or suggest), admit that (Fr. admettre, instead of acknowledge), affirm that (Fr. affirmer instead of assert or claim), establish that (Fr. établir, instead of demonstrate), underline that (Fr. souligner, instead of stress). This is confirmed by the fact that several of the French equivalents of these verbs are also found in the French RAs, namely affirmer, considerer, penser (think), souligner. While unlikely to cause any serious ambiguities, these lexical choices by FWE indicate that the collocational bundles that are a recurrent feature of academic discourse in English are in many cases unfamiliar to them.

\section{Identifying the Degree of Writer Commitment to the Cited Text}

One explanation for the relative scarcity of reporting verbs in the FWE drafts, mentioned above, is their greater use of introductory adverbials such as according to; another possible explanation is that they use instead the 'would-conditional' as a way of expressing their point of view. In this section we will explore these two hypotheses in the light of a number of recurrent problems observed with the use of according to and the would conditional form by the FWE.

In the absence of a reporting verb, the expression according to is one of the most common ways of introducing and identifying the source of information in English RAs. The generally recognized equivalent of according to in French is selon. A corpus search revealed however much wider recourse to French selon by the French writers (248 occurrences) than by the NS writers to according to (70 occurrences), with the FWE use lying interestingly between the two (110 occurrences). Expressed as frequencies per 10,000 words of running text, this works out as $10.1(\mathrm{~F}), 2.4(\mathrm{NS})$, and 5.8 (FWE) respectively. This perhaps suggests some differences in usage between the two expressions that are not necessarily widely reported in the literature.

\subsection{According to Versus Selon: Differing Degrees of Writer Commitment}

Two main values are traditionally associated with the expression according to (see for example Quirk et al. 1985; New Merriam Webster 1989), that of origin and that of conformity, as in (i) and (ii) below.

(i) According to Dr. Santos the cause of death was drowning (origin: as stated or as attested by)

(ii) Everything went according to plan (conformity: in accordance/conformity with) 
In the present discussion relating to citation practices it is the first meaning that we are principally concerned with. As Quirk et al. explain, when used in the sense of (i), according to signals not so much a reaction to but an interpretation of events by an outside source (Quirk et al. 1985; 712). The enunciator attributes entire responsibility for this interpretation or claim to the cited authority/source. Although obviously the reliability of the information will also depend on who/ what is cited as the source, the writer's own commitment to this point of view is not expressed. Example (4), from the NS subset, demonstrates this enunciative homogeneity:

(4) According to Lobeck (1995), verb phrase ellipsis is licensed by a head specified for strong agreement. (NS)

Through this choice of formulation the writer shows that she is not the source of the information and not therefore responsible for the reliability of the information or for any possible defects. The reported information is in the indicative (is licensed) and does not give any hint of the enunciator's own stance to the information transmitted.

In French, however, selon is used slightly differently. It can be used to express either full commitment to, or distance from, the cited source. This explains why in French it is possible to say 'selon moi', as well as selon $X^{2}$. There are several cases in the French RAs where selon is used with a first person pronoun:

(5) Le plus souvent, les EC sont catégorisées en EC «ponctuelles » et «duratives », ou encore en «EC-dates » et «EC-durées » (cf. Muller et al. 04) (...) Selon nous, cette distinction n'est pas pertinente. $(\mathrm{F})$

(EC are usually categorised as 'punctual' and 'durative' EC, or as 'EC-dates' and 'ECduration' (cf. Muller et al. 04) (...) According to us, this distinction is not a relevant one.)

In English, however, it is not usually possible to combine source and opinion in this way. The use of according to with a first person pronoun (according to me/us) is theoretically excluded (Bolinger 1990). ${ }^{3}$

The use of French selon thus appears more varied and flexible than that of according to in English in that it can be used in contexts where the writer wishes to remain neutral but can also express the writer's viewpoint on the cited source.

(6) Selon Costermans et Bestgen (1991), Segal et al. (1991), Zwaan (1996) entre autres, toutes les expressions temporelles n'ont pas la même efficacité. Nos analyses confirment cette these $(\mathrm{F})$

(According to Costermans and Bestgen (1991), Segal et al. (1991), Zwaan (1996) inter alia, not all temporal expressions are equally efficient. Our analyses confirm this)

\footnotetext{
2 The scope of such evidential expressions seems in fact to vary considerably from one language to another. In Italian the expression secondo can likewise be used with first person reference, secondo me. In Spanish, however, según me is, as in English, usually unacceptable in writing (John Swales, personal communication).

${ }^{3}$ First person references are occasionally possible for purposes of emphasis or contrast (see Bolinger 1990).
} 
(7) Selon Hasher et Zacks (1988), le vieillissement serait associé à un dysfonctionnement des processus attentionnels inhibiteurs (...). Il y aurait maintien en mémoire de travail d'informations distractives ... Or, en situation de conduite, les stimuli visuels qui se présentent au conducteur sont nombreux et cette altération pourrait avoir des répercussions négatives sur ... (F)

(According to Hasher and Zacks (1988), ageing would be associated with dysfunction of inhibitory attention processes (...) Distracting information would be retained in the working memory... In fact, when driving, the driver encounters a large number of visual stimuli and this dysfunction could have a negative impact on...)

In (6), French selon is used in much the same way as English according to. The enunciator attributes the statement to an outside source. Only in the second sentence does the writer's subsequent commitment to the source become clear. In (7) however the situation is rather different. Here, the source of the claim, Hasher and Zacks, is clearly identified by the use of selon; but the conditionals in the following two clauses also allow the citing writer to include her own point of view-i.e. lack of full commitment - towards the source. As the subsequent (3rd) sentence makes clear, the citing writer does not agree with the information reported by Hasher and Zacks and presents an alternative hypothesis. As Celle (2004) has shown, the association of French selon with a conditional can enable the writer to simultaneously express a double point of view-that of the cited source, and his own stance towards this source.

These subtle differences in use between the two evidential expressions in English and French can be problematic when moving from one language to another. In several cases in the drafts the French writer, under the influence of French selon, uses according to with a conditional verb, in order to emphasise his lack of commitment to the cited information. As in English, however, the use of according to automatically implies distance from the source, this combination of according to + the conditional results in passages such as (8) where there is a double (redundant) marking of writer distance which is not only linguistically incorrect but also obscures the writer's intended stance.

(8) According to Pinker (1984) semantic bootstrapping is the mechanism that allows children to determine which words fall into the category of noun or verb in their mother language. The discovery of noun and verb categories would depend on word meaning, which is acquired early and shaped by the child's interactions with her material and human environment. Children would thus start by constructing semantically appropriate representations of the linguistic items they are producing, and their representations would, in turn, help them grasp syntactic organisation (FWE).

\subsection{Use of the Conditional in French and English}

As Sect. 4.1. has already shown, the French conditional fulfills functions not easily transposable to English, and seems to be a major source of problems for FWE in our corpus. The following example from our draft corpus probably stems directly 
from the influence of French, and would we believe be very unclear for the English reader:

(9) Indeed, the semantics of early words is notoriously difficult to delineate (Bloom 1991), and verb semantics would be harder (Gleitman 1990; Golinkoff et al. 1995) - thus accounting for their later acquisition (Gentner 2006) (FWE).

In French the conditional allows the writer to report speech whilst at the same time dissociating herself from the cited source of information. It is extremely common in news discourse, particularly when the source of information is potentially unreliable or the report unconfirmed, as illustrated by the following headline (Le Monde, 6 Sept. 2011):

(10) Adnan Bakhour aurait été enlevé par des hommes armés le 29 août (A.B. is believed to have been kidnapped by armed men on 29th August)

As the suggested translation makes clear, this subjective modal value of the French conditional cannot be rendered in English by the equivalent verb form.

This use of the conditional is also extremely common in French research discourse, as it allows writers to report the cited findings or claims without necessarily committing themselves to their validity. A typical example from our French dataset is:

(11) Adjointes aux informations textuelles, ces connaissances seraient impliquées dans des activités complexes comme l'anticipation d'événements... (Graesser et al. 2002) (F).

(Combined with textual information, this knowledge would be involved in complex activities such as anticipating events)

The French conditional here is however intrinsically ambiguous, allowing two different interpretations: it can reflect either the original authors' (Graesser et al.) hedging of their claim, in which case the citing writer is simply reproducing their modalised statement, initially expressed in the conditional; or it can reflect the writer's intention not to commit herself to Graesser et al.'s claim. If the latter, there are two points of view expressed in a single clause: the claim made by Graesser et al., and the writer's marking of her distance from this claim through the use of the conditional. This double, or heterogeneous, enunciation that is possible with the French conditional raises similar problems for French writers to those discussed above concerning selon.

In English, the conditional cannot be used in this way and the writer is faced with a different set of choices. If the writer is neutrally reporting an initially tentative claim, this can be indicated either by using an attributive prepositional phrase such as according to with a modal expression in the main clause:

According to Graesser et al. this knowledge may be involved...

or by the choice of a reporting verb followed by an indicative:

Graesser et al. hypothesize that/speculate that this knowledge is involved... 
as the following example from our NS dataset shows:

(12) Katsos and Bishop hypothesised that low child performance in comprehension is an artefact of the task (NS)

If, on the contrary, the writer wishes to distance herself from the cited authors' claim, this can be indicated either by using according to with an indicative:

According to Graesser et al., this knowledge is involved...

or by using other lexical choices of reporting verbs that convey the writer's lack of commitment, again with an indicative:

Graesser et al. claim that/argue that this knowledge is involved...

The use of the indicative means that only one point of view is expressed in the reported clause, that of the cited authors. As Thompson and Ye (1991) point out, however, the evaluative potential of reporting verbs can in some cases introduce a certain ambiguity between the writer's interpretation of the cited authors' claim and the original claim. As Charles (2006: 325) comments:

We should note here, however, that the uncertainty expressed in the verb may be the 'writer's interpretation' in Thompson and Ye's terms (1991). It is attributed to the cited author by the writer and it is possible that the uncertainty is not present in the original text. It could be that writers attribute uncertainty to a cited author with whom they wish to disagree, since a lack of certainty on the part of the cited author would make the disagreement less face-threatening.

Due to these language-specific differences in the resources available for marking degrees of distance and/or commitment, the writer's positioning with respect to the cited sources is frequently unclear in the FWE drafts, where the double, or heterogeneous, enunciation expressed by the conditional in French has been directly transferred into English, resulting in passages such as (13):

(13) [Mannose] could come from polysaccharides of tissues, e.g. leaves... and would be used by a wide range of microorganisms (Wood and Stanway 2001) (FWE).

This type of formulation in fact deprives the original enunciators (Wood and Stanway) of their enunciative autonomy: their hypothesis or claim is 'de-asserted', or further modalised by the writer, making it difficult to distinguish between the two. It therefore has a negative impact on the clarity of the argument in English, given the different values of the conditional in the two languages. These languagespecific differences, we would argue, constrain the writer to selecting a suitable reporting verb + indicative when writing in English, rather than trying to combine the two points of view in a single clause. In the present case, the use of a reporting verb would have made it possible to attribute the claims unambiguously and make the respective positions of the original enunciators and of the writer clear (...Wood and Stanway (2001) argue that/consider that it is used...).

Even when a reporting verb is used, the French writers in many cases continue to use the conditional in the reported clause, in an attempt to express, as in French, their own stance towards this hypothesis-in this case, disagreement with Lyons: 
(14) John Lyons (1977) hypothesized that the abstract meaning of nouns would be derived from the 'words for persons, animals and things' prototype. But ontogenesis does not follow such a clear path,... (FWE)

This double marking of distance effectively cancels out the role of the reporting verb. A similar phenomenon was observed with according to (example (8) above). In cases such as these, the reader who is not familiar with the French language and with the subjective modal value of the conditional would probably have difficulty grasping the writer's intended meaning.

\section{Conceding Cited Claims: French si/English if}

A final problem with the expression of stance concerns concession. Concession is important in citation, as it allows the writer to take existing knowledge or others' claims into account while at the same time backgrounding them, in order to prioritise her own opinion or claim in the main clause, as illustrated by example (15) from the NS subset:

(15) Although there is some experimental work on adults' comprehension of over-informative expressions (Mangold and Pobel, 1988; Pechmann, 1989; Maes et al., 2004; Arts, 2004), there is scant research on this phenomenon in development (NS).

In (15), the research by the authors referred to in the brackets is efficiently backgrounded, paving the way for the gap in knowledge and creating the subsequent niche for the author's own claim.

There are however several cases in the drafts corpus where the concessive status of the cited information in cases like this is rather unclear. In (16), for example, the references are attributed unambiguously, but the author's stance does not clearly emerge.

(16) It is important to mention here that if projects for creating linguistic ontologies already exist (see [21][22] and [23] about the biomedical domain in particular), no one [sic] address the issue of creating an ontology of modality (FWE).

Is the information concerning the cited references hypothetical or asserted? The use of if here is rather disconcerting and appears to be a direct interference from French. In French the subordinator si has a wider range of values than English if and is frequently used not only to signal a conditional relation, but also to indicate a concessive one as in (17):

(17) La limitation de la dissection du curage axillaire au seul étage I, si elle diminue le taux de complications, n'apparaît pas être une alternative satisfaisante (F)

(Limiting axillary node dissection to the $\mathrm{T} 1$ stage, although it decreases the rate of complications, does not provide a satisfactory alternative)

In the French example (17), the author is not questioning whether or not the rate of complications has decreased. The information expressed in the conditional clause (or p clause) is actually the case. In the same way, in (16) above the FWE 
author does not intend to call into question the fact that projects for creating linguistic ontologies do already exist. In English, however, if is not generally used in this sense; the subordinator although or while is preferred when the reality expressed in the concessive clause is presupposed. Sentence (16) would have been far clearer for the reader with a concessive subordinator:

(16') It is important to mention here that although/whilst projects for creating linguistic ontologies already exist (see [21][22] and [23] about [...]

Likewise in the following extract, the French-influenced use of the concessive if-clause to contrast two sets of findings is confusing in English.

(18) If interesting developments concerning the interaction of syntactic, semantic/pragmatic and rhythmic cues in French phonological phrasing have been brought in the optimality theory framework a few years ago (see for French [6] and [7], among others), more recent works dealing with extra-sentential elements in spontaneous speech showed that the things were not as evident as one believed [8]. (FWE).

The replacement of if by although would have made the message far more rhetorically effective. Although arguably not a source of real ambiguity, such anomalies of usage are we contend disconcerting for the reader and can once again mask the writer's stance.

\section{Concluding Remarks}

Although an initial quantitative comparison between the uses made of citations in English research articles by expert French researchers as opposed to English researchers revealed few major differences, on closer scrutiny we have observed a number of linguistic features of FWE citation use that may adversely affect the overall rhetorical effectiveness and clarity of their articles. Considered in isolation some of these points may appear unimportant, but when viewed collectively we contend that they lead to subtle differences in positioning and commitment not always being communicated as effectively as they might be.

Four features were found to be particularly problematic for expert French writers of English in our corpus: reporting that-clauses, together with the use of according to, of the would conditional, and concessive if-clauses. The study has shown that expert FWE not only use reporting that-clauses far less frequently than their NS counterparts but also demonstrate a lack of lexical variety, and a certain lack of familiarity with the collocational bundles used in RAs with this structure. While some of these points bear a superficial resemblance to those highlighted by studies of novice researchers' citation practices (lack of lexical variety and inappropriate choice of the reporting verb, in particular, generally attributed to a lack of rhetorical mastery in the case of novices), the comparison with the French RA subset has shown that the explanation of these problems probably lies elsewhere in the case of expert FWE. The marked similarity between the use of reporting verbs 
in the FWE and F subsets, in terms of both frequency and lexical variety, indicates that it is the influence of French citation practices which predominates. Unfortunately, however, the impression made on the reader of the English text, who has different expectations, is that of an insufficient mastery of the nuances of positioning.

The second problem addressed, the use of according to, likewise shows some surface resemblances between novices and the expert FWE, in that in both cases the evidential adverbial is heavily used, but here again, on the basis of the corpus data, we would attribute this feature of FWE citation practice to the influence of the 'equivalent' expression in their native language, i.e. French selon. The problems here arise from the cross-linguistic differences in the degrees of writer commitment that can be expressed by according to and selon: the latter, unlike according to, can be used to express either full writer commitment to the cited source or distance from the source, and, when used with the conditional, to express a double enunciation, that of the cited source and the writer's own stance towards this source. The direct transfer of these features by FWE into their texts in English can result in ambiguity as to the writer's position towards the cited authors.

The influence of French citation practices can be perceived even more clearly in the FWE use of the would-conditional, with or without according to. The conditional is used extremely frequently in French academic discourse for hedging, since it enables the writer to report others' claims and results without committing herself, or to distance herself from the reported work. This value cannot be directly transferred into English, and necessitates reformulation via other linguistic means. We have observed, however, a recurrent tendency of the French writers of English to use would in the same way as the French conditional, which has a negative impact on the clarity of the argument, and have suggested that this use of the conditional may be one of the explanations for the underuse of reporting verbs by FWE.

The last feature examined here concerns concession, an important aspect of positioning for writers when citing other sources. Concession is frequently expressed in French RAs by subordinate si-clauses; again, however, as with the pair selon/according to, the range of values that French si and English if can take is not the same. When the reality expressed in the concessive clause is presupposed, English prefers to use subordinators such as although or while to express a concessive relation. In the FWE drafts, however, if-clauses are commonly used to contrast two points of view or concede a point; for the reader, this is confusing and obscures the writer's stance towards the cited information.

Citing may seem at first sight to be an aspect of research publications which is relatively culture-free: the systems used are international, and expert writers are familiar with journals' expectations. As this study has shown, however, citing in English, for writers of other languages, is far from straightforward. We have detected many instances where the influence of the French writers' native language and academic culture results in ambiguity as to the writer's stance and degree of commitment to her sources. Given the importance of citation in research articles, this can represent a major weakness in the rhetorical efficacy and clarity of the 
argument. It would be interesting to apply the approach adopted here to the study of citation practices by expert writers of other language origins who also have to publish in English, in order to pinpoint these often subtle but potentially critical issues.

\section{References}

Biber, D., Johansson, S., Leech, G., Conrad, S., \& Finegan, E. (1999). Longman grammar of spoken and written English. London: Longman.

Boch, F., \& Grossmann, F. (2002). Se référer au discours d'autrui: quelques éléments de comparaison entre experts et néophytes. Actes du colloque international, L'écrit dans l'enseignement supérieur (pp.41-51). Bruxelles: Enjeux.

Bolinger, D. (1990). According to. Journal of English Linguistics, 23, 225-238.

Carter-Thomas, S. (2007). The 'Iffiness' of medical research articles: A comparison of English if and French si. In K. Fløttum (Ed.), Language and discipline perspectives on academic discourse (pp. 150-175). Newcastle: Cambridge Scholars Publishing.

Celle, A. (2004). La Traduction du conditionnel journalistique: modes de construction de l'altérité énonciative en français, en anglais et en allemand. In J. M. Lopez Munoz, S. Marnette, \& L. Rosier (Eds.), Le Discours Rapporté dans tous ses Etats (pp. 500-509). Paris: L'Harmattan.

Charles, M. (2006). Phraseological patterns in reporting clauses used in citation: A corpus-based study of theses in two disciplines. English for Specific Purposes, 25, 310-331.

Charolles, M. (2005). Framing adverbials and their role in discourse cohesion: From connection to forward labelling. In M. Aurnague, M. Bras, A. Le Draoulec \& L. Vieu (Eds.), SEM-05 Proceedings, (pp.13-30). Biarritz.

Charolles, M., \& Péry-Woodley, M.P. (Eds.). (2005). Les adverbiaux cadratifs. Special issue of Langue française, p.148.

Chuquet, H., \& Paillard, M. (1987). Approche linguistique des problèmes de traduction anglaisfrançais. Paris: Ophrys.

Fløttum, K., Dahl, T., \& Kinn, T. (2006). Academic voices across languages and disciplines. Amsterdam: Benjamins.

Hyland, K. (1999). Academic attribution: Citation and the construction of disciplinary knowledge. Applied Linguistics, 20(3), 341-367.

Hyland, K. (2002). Activity and evaluation: Reporting practices in academic writing. In J. Flowerdew (Ed.), Academic Discourse (pp. 115-130). London: Longman.

Lillis, T., Hewings, A., Vladimirou, D., \& Curry, M. J. (2010). The geolinguistics of English as an academic lingua franca: Citation practices across English-medium national and Englishmedium international journals. International Journal of Applied Linguistics, 20, 111-135.

Mansourizadeh, K., \& Ahmad, U. K. (2011). Citation practices among non-native expert and novice scientific writers. Journal of English for Academic Purposes, 10, 152-161.

New Merriam-Webster Dictionary. (1989). Merriam-Webster Inc.

Quirk, R., Greenbaum, S., Leech, G., \& Svartvik, J. (1985). A comprehensive grammar of the English language. London: Longman.

Swales, J. M. (1986). Citation analysis and discourse analysis. Applied Linguistics, 7(1), 39-56.

Swales, J. M. (2004). Research genres. Cambridge: Cambridge University Press.

Swales, J. M., \& Feak, C. B. (2004). Academic writing for graduate students (2nd ed.). Ann Arbor: University of Michigan Press.

Swales, J. M., \& Feak, C. B. (2009). Telling a research story: Writing a literature review. Ann Arbor: University of Michigan Press. 
Thomas, S., \& Hawes, T. P. (1994). Reporting verbs in medical journal articles. English for Specific Purposes, 13, 129-148.

Thompson, G., \& Ye, Y. (1991). Evaluation in the reporting verbs used in academic papers. Applied Linguistics, 12, 365-382.

Thompson, P., \& Tribble, C. (2001). Looking at citations: Using corpora in English for academic purposes. Language, Learning and Technology, 5(3), 91-105. 